\title{
Scalar field perturbations in asymptotically Lifshitz black holes
}

\author{
Alex Giacomini, ${ }^{1}$ Gaston Giribet, ${ }^{2}$ Mauricio Leston, ${ }^{3}$ Julio Oliva, ${ }^{1}$ and Sourya Ray ${ }^{1}$ \\ ${ }^{1}$ Instituto de Ciencias Físicas y Matemáticas, Universidad Austral de Chile, Valdivia, Chile \\ ${ }^{2}$ Departamento de Física, Universidad de Buenos Aires FCEN-UBA y Conicet, Argentina \\ ${ }^{3}$ Instituto de Astronomía y Física del Espacio IAFE, UBA-Conicet, Argentina
}

(Received 9 March 2012; published 1 June 2012)

\begin{abstract}
We consider scalar field perturbations about asymptotically Lifshitz black holes with dynamical exponent $z$ in $D$ dimensions. We show that, for suitable boundary conditions, these Lifshitz black holes are stable under scalar field perturbations. For $z=2$, we explicitly compute the quasinormal mode frequencies, which result to be purely imaginary, and then obtain the damping-off of the scalar field perturbation in these backgrounds. The general analysis includes, in particular, the $z=3$ black hole solution of three-dimensional massive gravity.
\end{abstract}

DOI: 10.1103/PhysRevD.85.124001

\section{INTRODUCTION}

\section{A. Lifshitz holography}

It has been conjectured in [1] that the nonrelativistic Lifshitz-like fixed points admit a dual description in terms of holographic gravity models, mimicking what happens in the standard AdS/CFT correspondence for the case of relativistic conformal field theories [2]. The proposal in [1] is part of a more general program, whose goal is to generalize AdS/CFT correspondence to the area of condensed matter physics. The importance of having a tool to describe strongly-correlated condensed matter systems is undoubtable, and this is the reason why much attention has been focused on this area in the last four years.

In this context, a Lifshitz fixed point refers to a nonrelativistic system described by a field theory exhibiting anisotropic scale invariance under the transformation

$$
\vec{x} \rightarrow \lambda^{2} \vec{x}, \quad t \rightarrow \lambda^{2 z} t
$$

with $z \in \mathbb{R}_{>1}$ in the spacetime coordinates, but no Galilean invariance. Systems with such a behavior appear, for instance, in the description of strongly correlated electrons. These systems are characterized by the value of the dynamical exponent $z$ that parametrizes the scale transformation (1). The proposal in [1] is that, analogously to the case of relativistic conformal field theory, the $(D-1)$ dimensional Lifshitz fixed points would also admit a geometric dual description in $D$ dimensions. The geometric realization is such that the $D$-dimensional asymptotically Anti-de Sitter spaces of the standard $\mathrm{AdS}_{D} / \mathrm{CFT}_{D-1}$ picture get replaced by spaces whose metrics asymptote the geometry

$$
d s^{2}=-\frac{r^{2 z}}{l^{2 z}} d t^{2}+\frac{l^{2}}{r^{2}} d r^{2}+r^{2} d \vec{x}_{D-2}^{2}
$$

at large $r$, where $d \vec{x}_{D-2}^{2}$ is the metric of the $(D-2)$ dimensional plane $\mathbb{R}^{D-2}, l$ is a positive parameter with dimensions of length, and $z$ is a real parameter that ultimately coincides with the dynamical exponent of the scale
PACS numbers: 04.70.-s, 04.50.Gh, 04.60.Kz, 11.25.Tq

invariant theory that one is trying to holographically describe. Then, spaces (2), dubbed "Lifshitz spaces," happen to realize geometrically the scaling symmetry (1), which is certainly a symmetry of the metric above provided one also rescales the radial coordinate as $r \rightarrow \lambda^{-2} r$. In this generalized framework, the standard $\mathrm{AdS}_{D} / \mathrm{CFT}_{D-1}$ correspondence is recovered in the particular case $z=1$, for which (2) coincides with $\operatorname{AdS}_{D}$ in Poincare coordinates. This suggests a natural way of prescribing the holographic dictionary by mimicking the recipe of $[3,4]$ and adapting it to generic values of $z$. This holographic correspondence was thoughtfully studied, for instance, in references [5-10]. As seen in [1] and in its sequels, the fact of having $z \neq 1$ introduces new features in the boundary field theory correlators. For instance, the fact of having replaced AdS space by the Lifshitz space introduces a dependence of $z$ in the formula that gives the scaling dimension of the operators in the dual theory: In the case of a scalar field of mass $m$ in a $D$-dimensional Lifshitz space of dynamical exponent $z$, the scaling dimension $\Delta$ of the dual operator is given by the formula

$$
\Delta^{2}-(D+z-2) \Delta-m^{2} l^{2}=0,
$$

and its value corresponds to the asymptotic behavior of the bulk field at large distance, namely

$$
\Phi(x, t, r) \sim r^{-\Delta} \Phi_{0}(x, t)+\mathcal{O}\left(r^{-\Delta-1}\right)
$$

with the boundary configuration $\Phi_{0}(x, t)$ acting as a source for a dual field from the boundary theory point of view. Finiteness of the scalar field action on (2) demands

$$
2 \Delta>D+z-2,
$$

that is, it demands the following $z$-dependent generalization of the Breitenlohner-Freedman bound

$$
m^{2}>-\frac{(D+z-2)^{2}}{4 l^{2}} .
$$




\section{B. Correlation functions}

Correlation functions in the dual theory can then be computed by following the standard holographic prescription, which is required to calculate the on shell regularized Euclidean action with appropriate boundary asymptotic. To do this, one first performs a Wick rotation, defining $\tau=i t$. It is also convenient to define inverse radial coordinate as $u \equiv 1 / r$, for which the boundary turns out to be located at $u=0$. Then, one solves the scalar wave equation on the Euclidean background, whose solution, in the near boundary region, namely, for small values of $u$, behaves like

$$
\Phi(x, \tau, u) \sim u^{\Delta_{+}} \Phi_{0}^{(+)}(x, \tau)+u^{\Delta_{-}} \Phi_{0}^{(-)}(x, \tau),
$$

where $\Delta_{+}$are the solutions to (3), cf. (4).

To calculate the boundary two-point function one needs the bulk-boundary propagator. One writes the bulkfield configuration in terms of the Green function $G\left(x, \tau, u ; x^{\prime}, \tau^{\prime}, 0\right)$; namely,

$$
\Phi(x, \tau, u)=\int d \tau^{\prime} d x^{\prime} \Phi\left(x^{\prime}, \tau^{\prime}, 0\right) G\left(x, \tau, u ; x^{\prime}, \tau^{\prime}, 0\right) .
$$

In the momentum space the convolution simply reads

$$
\tilde{\Phi}(k, \omega, u)=\tilde{G}(k, \omega, u) \tilde{\Phi}(k, \omega, 0) .
$$

Then, one evaluates the bulk action, first integrating by parts and introducing an infrared cutoff. Namely,

$$
\begin{aligned}
S[\Phi]= & \int d x d \tau \int_{\epsilon}^{\infty} d u\left[-\Phi \partial_{\mu}\left(\sqrt{g} g^{\mu \nu} \partial_{\nu} \Phi\right)\right. \\
& \left.+\partial_{\mu}\left(\sqrt{g} g^{\mu \nu} \Phi \partial_{\nu} \Phi\right)\right] \\
= & \int d x d \tau\left[\sqrt{g} g^{u u} \Phi \partial_{u} \Phi\right]_{\epsilon}^{\infty} .
\end{aligned}
$$

In the momentum space, one finds

$$
S[\Phi]=\int d k d \omega[\tilde{\Phi}(k, \omega, 0) \mathcal{F}(k, \omega) \tilde{\Phi}(-k,-\omega, 0)]
$$

with the flux function $\mathcal{F}(k, \omega)$ being defined as follows:

$$
\mathcal{F}(k, \omega)=\left[\tilde{G}(-k,-\omega, u) \sqrt{g} g^{u u} \partial_{u} \tilde{G}(k, \omega, u)\right]_{\epsilon}^{\infty} .
$$

This eventually gives the boundary two-point function in the momentum space

$$
\mathcal{F}(k, \omega)=\langle O(k, \omega) O(-k,-\omega)\rangle,
$$

where only the flux factor evaluated on $\epsilon \rightarrow 0$ contributes, as the propagator $\tilde{G}(k, \omega, u)$ vanishes when $u \rightarrow \infty$. Nevertheless, the quantity one is interested in is the twopoint function in position space, which is obtained by Fourier transforming the expression above. This requires a careful treatment of the contact terms when Fourier is transforming the distributions [11] and taking the large separation limit.

In the particular case of strongly coupled $z=2$ Lifshitz points in $D-1=3$ dimensions at zero temperature, it was shown in [1] that the two-point function in the position space exhibits the form

$$
\left\langle O\left(x_{1}, t_{1}\right) O\left(x_{2}, t_{2}\right)\right\rangle \simeq \frac{c}{\left|x_{1}-x_{2}\right|^{2 \Delta}}+\ldots,
$$

where $c$ is a nonvanishing constant, $O(x, t)$ is an operator of the boundary theory, dual to the bulk operator $\Phi(x, t, r)$, and where the ellipses stand for contributions that are subleading in the large separation limit and contact terms. Notice that, in the case $D=4$ with $z=2$ and $m=0$, we have $\Delta=4$.

A remarkable feature of (14) is that it does not involve further suppressions coming from overall functions of the scale invariant quantity $\left|x_{1}-x_{2}\right|^{2} /\left|t_{1}-t_{2}\right|$ as one could have expected. As discussed in [1], such a suppression could have resulted in ultralocal behavior of the two-point function. Taking a closer look at the computation of (14) one observes that, in general, the reason why no ultralocal behavior is found in the boundary correlation function is the existence of nonanalytic contributions at small momenta. In the position space, such nonanalytic contributions yield a nonvanishing expression when $\left|x_{1}-x_{2}\right|>0$. If only positive integer powers of the momenta were present, then the Fourier transformation would give only contact terms.

\section{Finite temperature and the quasinormal modes}

Finite temperature regime, on the other hand, is also modified with respect to the standard $z=1$ case. We know from AdS/CFT that adding finite-temperature in the boundary conformal field theory corresponds to considering a black hole in AdS space. Similarly, in the case of anisotropic scale invariant theories, adding finitetemperature would correspond to considering in the bulk a black hole solution that asymptotes to (2) at large $r$.

The two-point function at finite-temperature is computed along the same lines as in the zero-temperature case: Basically, one solves the scalar field equation in the Euclidean black hole background imposing incomingmode boundary conditions at the horizon. Typically, the finite-temperature two-point function would exhibit an exponential damping-off produced by temperature effects. For instance, in $D=4$ and $z=2$ this was explicitly computed in [12], and the result was shown to have the form

$$
\left\langle O\left(x_{1}, t_{1}\right) O\left(x_{2}, t_{2}\right)\right\rangle \simeq \frac{e^{-\sqrt{4 \pi T}\left|x_{1}-x_{2}\right|}}{\left|x_{1}-x_{2}\right|^{3 / 2}}+\ldots
$$

Apart from being necessary to solve the field equation on the black hole background to compute the two-point function, the black hole quasinormal mode frequencies also have a direct interpretation from the boundary point of view: Considering a large black hole in the bulk corresponds to considering a thermal state in the dual boundary theory, and the decay of the scalar field in the bulk is directly associated to the decay of a perturbation of such 
a thermal state, whose relaxation time is given by the imaginary part of the quasinormal frequency $\omega(k),[13,14]$.

For the case of $z=2$ Lifshitz black holes, we will explicitly solve the quasinormal modes, analytically computing the frequencies and then obtaining the damping-off of the scalar field perturbations. Then, adapting the computation that holds for asymptotically AdS black holes to the case of generic $z$, we will also show that the imaginary part of the frequencies for the scalar field perturbation about the Lifshitz black hole backgrounds is always negative, proving the stability of these geometries under this kind of probe perturbations.

\section{LIFSHITZ BLACK HOLES}

In this section we will construct a higher-curvature gravity theory that admits $z=2$ Lifshitz black holes in arbitrary dimension $D$. We begin by considering the following ansatz:

$$
d s_{D}^{2}=-\frac{r^{2 z}}{l^{2 z}} F(r) d t^{2}+\frac{l^{2} d r^{2}}{r^{2} F(r)}+r^{2} d \vec{x}_{D-2}^{2},
$$

asking the metric function to obey $F(r \rightarrow+\infty) \rightarrow 1$ and have a simple root for $r>0$.

Analytic Lifshitz black hole solutions are not easy to find, and, consequently, constructing finite-temperature gravity duals has so far required the introduction of strange matter content whose physical interpretation and theoretical motivation are unclear. An alternative way of finding such a Lifshitz black hole solution is considering carefullytuned higher-curvature modifications to the EinsteinHilbert gravity action. This has been done, for instance, in Ref. [15-20]. Here, since we will be concerned with the Lifshitz black hole solutions with $z=2$, we will present the simplest higher-curvature gravity Lagrangians that admit asymptotically Lifshitz black holes with $z=2$ in arbitrary dimension $D$. As we will see, this amounts to considering terms in the gravity Lagrangian that are of cubic order in the Riemann tensor.

Then, we look for higher-curvature Lagrangians that admit metric (16) as solutions with $z \neq 1$. Since the most conservative generalization of Einstein gravity in higher dimensions, Lovelock theories, excludes static Lifshitz black holes [21], it is necessary to explore theories of gravity with higher-order field equations. ${ }^{1}$ In such a setup, black hole solutions have recently been reported, in particular, in the quadratic $D=3$ massive gravity of [22] a black hole with $z=3$ and $F(r)=1-r_{+}^{2} / r^{2}$ was found in Ref. [15]. In Ref. [17] the existence of such solutions in generic quadratic gravity theories in $D$ dimensions was explored. However, in the setup of [17] it is not possible to achieve the value $z=2$ for the dynamical exponent, and so

\footnotetext{
${ }^{1}$ The black holes and wormholes in vacuum, found in $[18,19]$ respectively, exist only for fixed values of the couplings of Lovelock gravity, at which Birkhoff's theorem are circumvented.
}

a natural next step is to include cubic terms in the Lagrangian.

We are interested in a theory that would result valid in $D=3$ as well, so that we consider only higher-curvature terms constructed with the Ricci tensor $R_{\mu \nu}$. In three dimensions, the Weyl tensor is zero and the Riemann tensor is fully determined in terms of its trace. Then, we only have two quadratic invariants, namely $\mathcal{R}_{(1)}^{2}=R^{2}$ and $\mathcal{R}_{(2)}^{2}=R^{\mu}{ }_{\nu} R^{\nu}{ }_{\mu}$, and three cubic invariants $\mathcal{R}_{(1)}^{3}=R^{3}$, $\mathcal{R}_{(2)}^{3}=R R_{\mu \nu} R^{\mu \nu}$, and $\mathcal{R}_{(3)}^{3}=R_{\nu}^{\mu} R_{\rho}^{\nu} R^{\rho}$. For $D \geq 7$, there are eight linearly independent, cubic scalars which can be constructed out of three Riemann tensors without involving its derivatives (see e.g. [23]). Here, for simplicity, we consider the simplest model defined by the action

$$
I=\int d^{D} x \sqrt{-g}\left(\sigma R-2 \Lambda+\sum_{i=1}^{2} b_{i} \mathcal{R}_{(i)}^{2}+\sum_{j=1}^{3} c_{i} \mathcal{R}_{(j)}^{3}\right)
$$

Already in $D=3$ one observes that this Lagrangian admits Lifshitz black hole solutions with $z=2$. More generally, one verifies that this Lagrangian happens to admit asymptotically Lifshitz black holes of the form (16) with $z=2$ for all values of $D$. Notice also that, since the action contains only traces of the Riemann curvature, this theory admits all Einstein manifolds as solutions, for which $R_{\mu \nu}=$ $\lambda g_{\mu \nu}$ with $\lambda$ being fixed in terms of the couplings. It is not hard to show that the field equations coming from (17) admit the following black hole metric as a solution:

$$
d s_{D}^{2}=-\frac{r^{4}}{l^{4}}\left(1-\frac{r_{+}^{2}}{r^{2}}\right) d t^{2}+\frac{l^{2} d r^{2}}{\left(r^{2}-r_{+}^{2}\right)}+r^{2} d \vec{x}_{D-2}^{2}
$$

provided the coupling constants $\Lambda, b_{i}$, and $c_{i}$ obey specific relations that we write in the Appendix. Here, the inverse of the Newton constant $\sigma$ and the parameter $l$ can be regarded as two arbitrary parameters. Theory (17) also admits Lifshitz black hole solutions with other values of $z$.

Black holes (18) present a Hawking temperature $T=$ $r_{+}^{2} /\left(2 \pi l^{3}\right)$. In the metric, $r_{+}$is an integration constant which represents the location of the event horizon $r=r_{+}$. This integration constant is an actual physical parameter provided at least one of the flat directions is compactified, which results in breaking the scaling symmetry (1). On the contrary, if all the flat directions are noncompact, then $r_{+}$ can be settled to 1 by a simple change of coordinates.

Having given a particular example of a theory that admits $z=2$ Lifshitz black holes in arbitrary dimensions, we can proceed and study the scalar response on these backgrounds.

\section{QUASINORMAL MODES}

\section{A. Quasinormal modes for $z=2$ black holes}

As pointed out in [12], the scalar field response in backgrounds (18) turn out to be exactly solvable in terms of 
hypergeometric functions. This is relevant, for instance, to compute boundary correlation functions. This is precisely the computation done in Ref. [12], where the finitetemperature two-point function was shown not to exhibit ultralocal behavior either. Here, we are interested in a different problem, namely, that of computing quasinormal mode frequencies on background (18). To solve this, we consider a massive scalar field on the black hole metric (18)

$$
\left(\square-m^{2}\right) \Phi=\frac{1}{\sqrt{-g}} \partial_{\mu}\left(\sqrt{-g} g^{\mu \nu} \partial_{\nu} \Phi\right)-m^{2} \Phi=0,
$$

and assume separability, namely,

$$
\Phi(\vec{x}, t, r)=e^{-i \omega t+i \vec{k} \cdot \vec{x}} R(r),
$$

where $\vec{k}$ is in principle an arbitrary vector. Quasinormal modes would come from imposing boundary conditions for the scalar field configuration at the horizon and at the boundary: We ask for incoming-mode boundary conditions at $r=r_{+}$while we ask the field to vanish at $r=\infty$. To implement these conditions, it is useful to consider the following coordinate transformation:

$$
y=\frac{r^{2}-r_{+}^{2}}{r^{2}}
$$

for which the range $r=\left[r_{+},+\infty\right)$ is mapped to $y=[0,1)$. This coordinate transformation induces a change of variable in the equation for the radial part of the scalar field $R(r) \rightarrow$ $R(y)$, which now has to fulfill the following equation:

$$
\frac{d^{2} R(y)}{d y^{2}}+\frac{(2+(D-4) y)}{2(1-y) y} \frac{d R(y)}{d y}+\frac{l^{2}\left(\omega^{2} l^{4}+\left(\omega^{2} l^{4}+k^{2} r_{+}^{2}\right) y^{2}-\left(2 \omega^{2} l^{4}+k^{2} r_{+}^{2}+m^{2} r_{+}^{4}\right) y\right)}{4 r_{+}^{4} y^{2}(1-y)^{2}} R(y)=0,
$$

where $k^{2}=\vec{k} \cdot \vec{k}$. This equation can be transformed into a hypergeometric equation, whose solutions for $i \omega l^{3} / r_{+}^{2} \notin$ $\mathbb{Z}$ read

$$
\begin{aligned}
R(y)= & y^{\alpha}(1-y)^{\beta}\left[A_{12} F_{1}(a, b, c, y)\right. \\
& \left.+A_{2} y^{1-c}{ }_{2} F_{1}(b-c+1, a-c+1,2-c, y)\right],
\end{aligned}
$$

where $A_{1}$ and $A_{2}$ are integration constants, ${ }_{2} F_{1}(y)$ stands for the hypergeometric function, and

$$
\alpha=-\frac{i \omega l^{3}}{2 r_{+}^{2}}, \quad \beta=\frac{D+\sqrt{D^{2}+4 m^{2} l^{2}}}{4},
$$

where the arguments of the hypergeometric function are

$$
\begin{aligned}
& a= \frac{1}{4 r_{+}^{2}}\left[\left(2+\sqrt{D^{2}+4 m^{2} l^{2}}\right) r_{+}^{2}-2 i \omega l^{3}\right. \\
&\left.+\sqrt{(D-2)^{2} r_{+}^{4}-4 l^{2}\left(k^{2} r_{+}^{2}+\omega^{2} l^{4}\right)}\right], \\
& b= \frac{1}{4 r_{+}^{2}}\left[\left(2+\sqrt{D^{2}+4 m^{2} l^{2}}\right) r_{+}^{2}-2 i \omega l^{3}\right. \\
&\left.-\sqrt{(D-2)^{2} r_{+}^{4}-4 l^{2}\left(k^{2} r_{+}^{2}+\omega^{2} l^{4}\right)}\right], \\
& c=1-\frac{i \omega l^{3}}{r_{+}^{2}} .
\end{aligned}
$$

One can use properties of the hypergeometric functions to express the solution as a linear combination of functions that are regular in other points. We prefer to use the basis above in which the problem of recognizing the ingoing mode at the horizon is simpler.

Imposing ingoing boundary condition at the horizon, which in these coordinates is located at $y=0$, requires
$A_{2}$ to vanish, since around the horizon the scalar field behaves as

$$
\begin{aligned}
\Phi & \sim_{y \rightarrow 0} A_{1} e^{-i \omega t} y^{-i g \omega}+A_{2} e^{-i \omega t} y^{i g \omega} \\
& \sim_{y \rightarrow 0} A_{1} e^{-i \omega(t+g \ln y)}+A_{2} e^{-i \omega(t-g \ln y)},
\end{aligned}
$$

where $g:=l^{3} / 2 r_{+}^{2}$, and where we have used the fact that ${ }_{2} F_{1}(a, b, c, 0)=1$. Therefore, setting $A_{2}=0$ gives the desired behavior, namely, ingoing modes at the horizon. One is then left with the following solution for the radial part:

$$
R(y)=A_{1} y^{\alpha}(1-y)_{2}^{\beta} F_{1}(a, b, c, y) .
$$

In order to impose a boundary condition at infinity, $y \rightarrow 1$, it is convenient to use the Kummer's identities for the hypergeometric function and express ${ }_{2} F_{1}(y)$ in terms of combinations of ${ }_{2} F_{1}(1-y)$. After performing this transformation the function (28) reads

$$
\begin{aligned}
R(y)= & A_{1} y^{\alpha}(1-y)^{\beta}\left[\xi_{12} F_{1}(a, b, a+b+1-c, 1-y)\right. \\
& +\xi_{2}(1-y)^{c-a-b}{ }_{2} F_{1}(c-a, c-b, 1+c \\
& -a-b, 1-y)]
\end{aligned}
$$

with

$\xi_{1}=\frac{\Gamma(c) \Gamma(c-a-b)}{\Gamma(c-a) \Gamma(c-b)} \quad$ and $\quad \xi_{2}=\frac{\Gamma(c) \Gamma(a+b-c)}{\Gamma(a) \Gamma(b)}$.

Now, we impose Dirichlet boundary conditions at infinity: Expanding the expression (29) when $y \rightarrow 1$, one has the following leading-order contributions coming from each of the terms inside of the square bracket of (29): 


$$
\begin{gathered}
\Phi \sim_{y \rightarrow 1} \xi_{1}(1-y)^{\beta}+\xi_{2}(1-y)^{\beta+c-a-b} \\
\sim_{r \rightarrow+\infty} \xi_{1} r^{-\Delta_{+}}+\xi_{2} r^{-\Delta_{-}}
\end{gathered}
$$

with

$$
\Delta_{ \pm}:=\frac{D \pm \sqrt{D^{2}+4 m^{2} l^{2}}}{2} .
$$

Notice that this gives the solutions to Eq. (3). For $m^{2} \geq 0$ the contribution given by $r^{-\Delta_{-}}$diverges at infinity, and then in order to impose Dirichlet boundary condition $\Phi \sim_{y \rightarrow 1} 0$, one is forced to impose

$$
\xi_{2}=\frac{\Gamma(c) \Gamma(a+b-c)}{\Gamma(a) \Gamma(b)}=0 .
$$

This is fulfilled provided $a=-n$ or $b=-n$ with $n \in$ $\mathbb{Z}_{\geq 0}$ with $c-a-b \notin \mathbb{Z}$. These conditions give rise to the following spectrum:

$$
\omega=-\frac{i}{l^{3}} \frac{l^{2} k^{2}+r_{+}^{2}\left(D+m^{2} l^{2}+4 n(n+1)+(2 n+1) \sqrt{D^{2}+4 m^{2} l^{2}}\right)}{\sqrt{D^{2}+4 m^{2} l^{2}}+2(2 n+1)},
$$

where we have reinserted $r_{+}$. In terms of the dimension $\Delta_{ \pm}$this reads

$$
\omega=-\frac{i}{l^{3}} \frac{l^{2} k^{2}+r_{+}^{2}\left[\Delta_{-}\left(\Delta_{-}-2(2 n+1)-D\right)+2(2 n+D)(n+1)\right]}{2(2 n+1)+D-2 \Delta_{-}} .
$$

In the particular case $D=4$, the frequencies (33) coincide with frequencies $\omega_{1}$ of Ref. [24].

At the special points $c-a-b \in \mathbb{Z}$, that is when $\sqrt{D^{2}+4 m^{2} l^{2}} / 2 \in \mathbb{Z}$, the solutions of hypergeometric equation develop logarithmic dependence. For $m^{2}>0$, the frequencies (33) are purely imaginary and, besides, the imaginary part is strictly negative. This implies that there are no oscillatory modes and the field is purely damped. In this case the leading term in the asymptotic behavior of the scalar field is given by $\Phi \sim_{y \rightarrow 1} r^{-\Delta_{+}}$, that we will call strong asymptotic behavior.

Notice that for $-(D / 2)^{2} \leq m^{2} l^{2}<0$ both $\Delta_{ \pm}>0$, and so both branches $r^{-\Delta_{ \pm}}$, fulfill the Dirichlet boundary condition with a continuous spectrum. This is similar to the case of AdS spacetime [25] for $m_{\mathrm{BF}}^{2} l^{2} \leq m^{2} l^{2}<0$, where $m_{\mathrm{BF}}^{2}:==-(D-1)^{2} /(2 l)^{2}$ stands for the BreitenlohnerFreedman mass [26]. Notice also that the bound $-(D / 2)^{2} \leq m^{2} l^{2}$ in the asymptotically Lifshitz black hole with $z=2$, corresponds to the AdS-BreitenlohnerFreedman bound in dimension $D+1$.

\section{B. Scalar field and Lifshitz soliton}

In three dimensions, the metric (18) allows one to construct a soliton by performing the following Wick rotation both in the spatial and the time coordinates; namely,

$$
x \rightarrow \frac{i l}{r_{+}} \tilde{t} \text { and } t \rightarrow \frac{i l^{3}}{r_{+}^{2}} X .
$$

The change in the radial coordinated $r=r_{+} \cosh \rho$ in the three-dimensional version of (18), supplemented by the double Wick rotation (35), gives the soliton metric

$$
d s^{2}=l^{2}\left[-\cosh ^{2} \rho d \tilde{t}^{2}+d \rho^{2}+\cosh ^{2} \rho \sinh ^{2} \rho d X^{2}\right],
$$

where $\rho \geq 0$, and the horizon of the black hole is mapped to the center of the everywhere regular soliton.
Asymptotically, this metric has an anisotropic scaling symmetry with $z=z_{\text {soliton }}=1 / 2$, and its Euclidean continuation is diffeomorphic to the Euclidean continuation of the black hole. Given the smoothness of the solution (36) and its lack of integration constants, it would be natural to regard this spacetime as a ground state. Some of the implications of this in the case $z=3$ have been already explored in [27], where it was shown that considering as a background the soliton constructed out from the $z=3$ black hole in three-dimensional massive gravity, it is possible to give account of the entropy of the black hole by means of a generalized version of Cardy's formula.

It is, therefore, natural to raise the question about the stability of the solitons constructed in this manner. In particular, if one considers a scalar field perturbations on the soliton (36), it is possible to integrate the field configurations in terms of hypergeometric functions. Then, by imposing regularity at the origin $(\rho=0)$ and vanishing field at infinity $(\rho \rightarrow \infty)$, one finds the normal modes of the field on the soliton. Nevertheless, it is possible to avoid this computation since requiring ingoing boundary condition at the horizon of the $z=2$ black hole (18) in three dimensions corresponds to requiring regularity of the scalar field at the origin of the soliton (36), and therefore the normal modes on the soliton can be obtained directly from the double Wick rotated quasinormal frequencies (33) on the black hole in three dimensions. ${ }^{2}$ This is, performing the changes

$$
k \rightarrow \frac{r_{+}}{l} i \omega_{\mathrm{sol}} \quad \text { and } \quad \omega \rightarrow \frac{r_{+}}{l^{3}} i k_{\mathrm{sol}},
$$

for $D=3$ in (33), and then solving for the frequencies on the soliton $\omega_{\text {sol }}$, one gets the following spectrum:

\footnotetext{
${ }^{2}$ The same occurs for Bañados-Teitelboim-Zanelli where the soliton obtained after the Wick rotation is $\mathrm{AdS}_{3}$.
} 


$$
\begin{aligned}
\omega_{\text {sol }}= & \pm\left[\left(2 n+\left|k_{\text {sol }}\right|+1\right) \sqrt{9+4 m^{2} l^{2}}+3+m^{2} l^{2}\right. \\
& \left.+4 n\left(n+\left|k_{\text {sol }}\right|+1\right)+2\left|k_{\text {sol }}\right|\right]^{1 / 2},
\end{aligned}
$$

where $n \in \mathbb{Z}_{\geq 0}$, and $k_{\text {sol }}$ is the momentum along the $X$ direction in (36).

Note that the normal frequencies in (38) are real provided $m^{2}>-9 /\left(4 l^{2}\right)$, so the stability of the scalar field propagation on the soliton is guaranteed on this range.

\section{Scalar field stability for generic $z$}

We observe from (33) that the imaginary part of the frequency is actually negative, namely, $\operatorname{Im}(\omega)<0$. As we will show below, this result could have been shown by a more general argument, which comes from adapting an argument of [13], valid for asymptotically $\mathrm{AdS}_{D}$ black holes, to generic values of $z$. More precisely, having $\operatorname{Im}(\omega)<0$ for Dirichlet boundary conditions is a generic feature of the asymptotically Lifshitz black holes of the form (16) with ${ }^{3} F(r)=1-r_{+}^{2} / r^{2}$, and for arbitrary values of the dynamical exponent $z$. To see this, let us consider the ansatz

$$
d s_{D}^{2}=-\frac{r^{2 z}}{l^{2 z}} F(r) d t^{2}+\frac{l^{2} d r^{2}}{r^{2} F(r)}+r^{2} d \Sigma_{D-2}^{2},
$$

with $d \Sigma_{D-2}^{2}$ being the metric on the $(D-2)$-dimensional base manifold. By a simple change of variables, metric (39) can be rewritten as follows:

$$
d s_{D}^{2}=-r^{2 z} F(r) d v^{2}+2 r^{z-1} d v d r+r^{2} d \Sigma_{D-2}^{2},
$$

where we have fixed $l^{2}=1$ for short.

Now, consider again the scalar field Eq. (19) and propose the following form for its solution:

$$
\Phi=e^{-i \omega v} R(r) Y(\Sigma),
$$

where $Y(\Sigma)$ is a harmonic function on $\Sigma$, i.e. $\tilde{\nabla}_{\Sigma}^{2} Y(\Sigma)=$ $-k^{2} Y(\Sigma)$, where $\tilde{\nabla}_{\Sigma}^{2}$ is the Laplacian intrinsically defined on $\Sigma_{D-2}$, in our case $\Sigma_{D-2} \subseteq \mathbb{R}^{D-2}$. Using this, one obtains

$$
\begin{aligned}
& Y(\Sigma)\left[-i \omega r^{1-z} \frac{d R}{d r}+r^{3-D-z} \frac{d}{d r}\left(F r^{D+z-1} \frac{d R}{d r}-i \omega r^{D-2} R\right)\right. \\
& \left.-\left(k^{2} r^{D+z-5}+m^{2}\right) R\right]=0 .
\end{aligned}
$$

If one further defines $R(r)=r^{2-D / 2} \psi(r)$, this equation becomes

$$
\frac{d}{d r}\left(\frac{f(r)}{r^{z-1}} \frac{d \psi}{d r}\right)-2 i \omega \frac{d \psi}{d r}-V(r) \psi=0,
$$

where $f(r)=r^{2 z-2}\left(r^{2}-1\right)$ with $r_{+}=1$, and where the effective potential is

\footnotetext{
${ }^{3}$ The same argument works without assuming the special form $F(r)=y=1-r_{+}^{2} / r^{2}$ but merely requiring $F^{\prime}(r)>0$ outside the horizon.
}

$$
\begin{aligned}
V(r)= & \frac{1}{4 r^{z+1}}\left((D-2)(D-6+2 z) r^{2 z-2}\left(r^{2}-1\right)\right. \\
& \left.+4\left((D-2)+m^{2}\right) r^{2 z}+4 k^{2} r^{2 z-2}\right) .
\end{aligned}
$$

Notice that for $z \geq 2$ this potential is strictly positive outside the black hole. Notice also that in the case $z=1$, this potential is positive for $r \geq 1$.

By manipulating Eq. (42) and integrating by parts one can show that the following equation holds:

$$
\int_{r=1}^{\infty} d r\left(\frac{f(r)}{r^{z-1}}\left|\frac{d \psi}{d r}\right|^{2}+V(r)|\psi|^{2}\right)=-\frac{|\omega|^{2}\left|\psi_{(r=1)}\right|^{2}}{\operatorname{Im}(\omega)},
$$

where Dirichlet boundary conditions for the scalar field were imposed at infinity. Condition (5) guarantees the convergence of the left-hand side of (44). Then, since the left-hand side of (44) is strictly positive, it demands $\operatorname{Im}(\omega)<0$, and then we conclude the stability of the scalar under perturbations respecting these boundary conditions, provided the generalized Breitenlohner-Freedman bound (6) is obeyed. This analysis includes, in particular, the $z=3$ black hole solution of three-dimensional massive gravity, whose quasinormal modes were recently studied in [28].

\section{CONCLUSIONS}

In this paper we have studied scalar field perturbations in asymptotically Lifshitz black holes with dynamical exponent $z=2$ in arbitrary dimension $D$. These solutions appear, for instance, as exact solutions of higher-curvature theories of gravity, which consist of augmenting EinsteinHilbert action with terms that are quadratic and cubic in the Riemann tensor.

For these black holes, we have explicitly solved the quasinormal modes for scalar field perturbations that obey suitable boundary conditions. We have computed the frequencies of these perturbations explicitly, finding that they are purely imaginary, with negative imaginary part. This implies the stability of these geometries under scalar field perturbations that respect the mentioned boundary conditions. The stability of these Lifshitz black holes under scalar field perturbations, on the other hand, can be anticipated by a more general argument: By adapting a computation previously done for asymptotically $\operatorname{AdS}_{D}$ black holes, we gave a concise argument that proves the stability of a more general class of black holes with arbitrary value of $z$ provided the generalized BreitenlohnerFreedman bound is satisfied.

By performing double Wick rotations of the black hole (18), we have constructed an everywhere regular solitonic solution in three dimensions. This solution has an asymptotically anisotropic symmetry. We have obtained the normal frequencies for the scalar field on this metric by performing a double Wick rotation of the quasinormal spectrum of the black hole. This is consistent since asking for an ingoing boundary condition for the scalar field on 
the horizon of the black hole corresponds to asking for regularity of the field at the center of the soliton. The spectrum obtained in this way shows that the scalar field oscillates without neither any damping nor exponential grow, provided the generalized BF bound is fulfilled. This analysis can be extended to higher dimensions, where in order to construct a soliton one must single out one of the flat directions on the metric (18), and then apply a double Wick rotation between the selected coordinate and time. Performing the corresponding Wick rotation on the quasinormal spectrum (33) itself in arbitrary dimensions gives the normal modes for the scalar perturbation on the soliton with frequencies that are real provided the generalized BF bound is fulfilled. It would be interesting to see if the nonlinear instability of AdS under the collapse of a backreacting scalar field [29] also appears in the case of the asymptotically Lifshitz backgrounds.

Before concluding, we would like to emphasize that in this article we have only considered the quasinormal mode computation for scalar fields, and even when from the holographic point of view such computation is interesting by its own right, concluding stability of asymptotically Lifshitz spacetimes would also need to consider, in particular, spin- 2 excitations. Being a higher-curvature theory, computing spin-2 perturbations of an action like (17) about an asymptotically Lifshitz geometry is certainly cumbersome, and since the equations of motion are of fourthorder, the results would, in general, exhibit ghosts. Still, particular higher-curvature models like the one introduced in [22] could likely result to be free of ghosts about Lifshitz black hole solutions, as well. This is an interesting question that requires further study.

\section{ACKNOWLEDGMENTS}

The work of A.G., J.O. and S.R. is supported by FONDECYT Grants Nos. 1110167, 11090281, 11110176, and CONICYT 791100027. The work of G. G. and M. L. is supported by PICT, PIP, and UBACyT grants from ANPCyT, CONICET and UBA, Argentina. The authors thank Pedro Álvarez, Eloy Ayón-Beato, Francisco Correa, and Bertha Cuadros-Melgar for comments.

After our paper appeared in arXives, we were informed by Oscar I. Fuentealba Murillo and his collaborators that, in an unpublished paper, he and collaborators performed computations similar to those of our Sec.III B

\section{A. APPENDIX}

The coupling constants of action (17) for the theory to admit $z=2$ Lifshitz black holes (16) in arbitrary dimension $D$ are given by

$$
\begin{gathered}
\Lambda=-\frac{\sigma l^{-2}}{3 C_{D}} D\left(3 D^{6}-26 D^{5}+107 D^{4}-20 D^{3}\right. \\
\left.-80 D^{2}+368 D+208\right), \\
b_{1}=\frac{\sigma l^{2}}{C_{D}}\left(3 D^{4}-15 D^{3}+6 D^{2}+104 D+32\right), \\
b_{2}=-\frac{\sigma l^{2}}{C_{D}(D+2)}(D-1)\left(D^{5}-D^{4}-20 D^{3}\right. \\
\left.+108 D^{2}+208 D+64\right), \\
c_{1}=-\frac{\sigma l^{4}}{3 C_{D}}(3 D+2)(D-8)(D-1)^{2}, \\
c_{2}=\frac{2 \sigma l^{4}}{C_{D}}\left(D^{3}-13 D^{2}+8 D+4\right), \\
c_{3}=\frac{8 \sigma l^{4}}{3 C_{D}}(4 D+1),
\end{gathered}
$$

where $\quad C_{D}:=D^{6}-8 D^{5}+53 D^{4}-130 D^{3}+124 D^{2}+$ $296 D+64$, which has no integer roots.
[1] S. Kachru, X. Liu, and M. Mulligan, Phys. Rev. D 78, 106005 (2008).

[2] J. Maldacena, Adv. Theor. Math. Phys. 2, 231 (1998).

[3] E. Witten, Adv. Theor. Math. Phys. 2, 253 (1998).

[4] S. Gubser, I. Klebanov, and A. Polyakov, Phys. Lett. B 428, 105 (1998).

[5] S. Ross, Classical Quantum Gravity 28, 215019 (2011).

[6] S. Ross and O. Saremi, J. High Energy Phys. 09 (2009) 009.

[7] T. Griffin, P. Horava, and C. Melby-Thompson, arXiv:1112.5660.

[8] M. Baggio, J. de Boer, and K. Holsheimer, J. High Energy Phys. 01 (2012) 058.
[9] R. Mann and R. McNees, J. High Energy Phys. 10 (2011) 129.

[10] M. Baggio, J. de Boer, and K. Holsheimer, arXiv:1112.6416.

[11] M. J. Lighthill, An Introduction to Fourier Analysis and Generalized Functions, Cambridge Monographs on Mechanics (University Press, Cambridge, 1958).

[12] K. Balasubramanian and J. McGreevy, Phys. Rev. D 80, 104039 (2009).

[13] G. Horowitz and V. Hubeny, Phys. Rev. D 62, 024027 (2000).

[14] D. Birmingham, I. Sachs, and S. Solodukhin, Phys. Rev. Lett. 88, 151301 (2002). 
[15] E. Ayón-Beato, A. Garbarz, G. Giribet, and M. Hassaïne, Phys. Rev. D 80, 104029 (2009).

[16] R-G. Cai, Y. Liu, and Y-W. Sun, J. High Energy Phys. 10 (2009) 080.

[17] E. Ayón-Beato, A. Garbarz, G. Giribet, and M. Hassaïne, J. High Energy Phys. 04 (2010) 030.

[18] M. Dehghani and R. Mann, J. High Energy Phys. 07 (2010) 019.

[19] J. Matulich and R Troncoso, J. High Energy Phys. 10 (2011) 118.

[20] W. Brenna, M. Dehghani, and R. Mann, Phys. Rev. D 84, 024012 (2011).

[21] R. Zegers, J. Math. Phys. (N.Y.) 46, 072502 (2005).

[22] E. A. Bergshoeff, O. Hohm, and P. K. Townsend, Phys. Rev. Lett. 102, 201301 (2009).
[23] S. Fulling, R. King, B. Wybourne, and C. Cummins, Classical Quantum Gravity 9, 1151 (1992).

[24] P. A. González, J. Saavedra, and Y. Vásquez, arXiv:1201.4521.

[25] D. Birmingham, I. Sachs, and S. N. Solodukhin, Phys. Rev. Lett. 88, 151301 (2002).

[26] P. Breitenlohner and D.Z. Freedman, Phys. Lett. B 115, 197 (1982).

[27] H. A. González, D. Tempo, and R. Troncoso, J. High Energy Phys. 11 (2011) 066.

[28] B. Cuadros-Melgar, J. de Oliveira, and C. Pellicer, Phys. Rev. D 85, 024014 (2012).

[29] J. Jalmuzna, A. Rostworowski, and P. Bizon, Phys. Rev. D 84, 085021 (2011). 